\title{
La organización temática de los recursos de información en las bibliotecas: somero análisis diacrónico desde la antigüedad hasta nuestros días
}

\author{
The Subject Organization of Information Resources in Libraries: a Brief Diachronic Analysis \\ from Antiquity to the Present
}

\author{
Adriana Suárez Sánchez \\ Universidad Nacional Autónoma de México, México \\ http://orcid.org/0000-0003-0215-337X
}

Recibido: 24 de julio de 2020

Aceptado: 03 de diciembre de 2020

Publicado: 21 de mayo de 2021

\begin{abstract}
Resumen
La organización temática de los recursos de información en las bibliotecas ha sido una actividad sustancial que ha prevalecido desde las civilizaciones antiguas hasta nuestros días. El objetivo de este trabajo es trazar una revisión diacrónica de la actividad, considerando los recursos, el proceso, las personas que lo desarrollan y los lenguajes de indización auxiliares. La metodología empleada consistió en el análisis de un conjunto de fuentes de información académicas sobre el tema. Se encontró que la organización temática de los recursos de información ha pasado por diversas etapas, englobadas en cuatro periodos: (1) antes del establecimiento de la bibliotecología como disciplina, (2) la estandarización bibliotecaria de los siglos XIX y XX, (3) los tiempos de la segunda mitad del siglo XX y (4) los albores del siglo XXI. Se concluye que el ordenamiento temático de los recursos de información es una actividad en constante evolución, a partir de los recursos de información, las tecnologías para su arreglo y las necesidades de la población usuaria.
\end{abstract}

\section{Palabras clave}

Organización temática, Indización, Catalogación temática, Acceso temático, Bibliotecas

\begin{abstract}
The subject organization of informational resources in libraries has been an essential activity since the ancient civilizations to our present day. The target of this work is to present a diachronic revision of the subject organization taking into consideration the resources, the processes, the people who develop them, and the indexing languages employed in such activities. The methodology for this work consists in the revision and analysis of a set of academic sources of information on the topic. This work proves that the subject organization of informational resources has undergone different phases that can be categorized into four different periods: (1) before the establishment of librarianship as a discipline, (2) the stage under the library codes and standards in the nineteenth and twenty centuries, (3) during the second half of the twenty century and (4) the starting of the 21st century. Conclusively, this is an activity that has evolved, constantly, as the informational resources, the technologies employed for their classification, and the needs of their users have required it.
\end{abstract}

\section{Keywords}

Subject organization, Indexing, Subject cataloging, Subject Access, Library 


\section{Introducción}

El acceso temático a los recursos de información es una opción de consulta que las bibliotecas han ofrecido desde antaño y se ancla en las necesidades de información de las personas usuarias. En la búsqueda de recursos es común que los usuarios tengan un autor o título en mente, pero también es frecuente que revelen una exigencia tópica asociada a su contexto académico, laboral, económico, familiar, recreativo, etc., por ejemplo, un estudiante que investiga sobre las "células procariontes", un trabajador social que busca información sobre "derechos infantiles" o un ama de casa que requiere la receta del "pastel tres leches".

Para cumplir con tal exigencia, las bibliotecas han desarrollado actividades dirigidas a la organización temática de los recursos de información (OTRI). Según Barité (2015), la OTRI es el proceso de descripción y representación del contenido temático de un documento, mediante un número limitado de términos tomados directamente del documento (palabras clave) o de vocabularios controlados (descriptores o encabezamientos de materia) que caracterizan al recurso, de modo que faciliten su futura recuperación.

Es una actividad que el personal bibliotecario realiza día a día; sin embargo, en medio de las múltiples ocupaciones laborales, pocas veces nos detenemos a reflexionar sobre su origen, establecimiento y funcionalidad. ¿Cuándo empezó la actividad? ¿En qué momento se instauró como proceso esencial de las bibliotecas? ¿Cómo evolucionó a través del tiempo? ¿Siempre han existido bibliotecarios encargados de la organización temática? ¿Siempre se ha desarrollado el proceso que realizamos actualmente? ¿Cuándo aparecieron los lenguajes de indización que apoyan la actividad?

La organización temática de los recursos de información desde una perspectiva diacrónica ha sido, principalmente, estudiada por autores de habla inglesa como Cleveland y Cleveland en su obra Introduction to indexing and abstracting (2013) donde ofrecen una visión panorámica que contempla su origen en las culturas antiguas, el proceso durante la Edad Media, la organización temática de los recursos después del surgimiento de la imprenta, el preludio de los tiempos modernos y los retos actuales caracterizados por las tecnologías de la información. De igual manera, también en lengua inglesa, se cuenta con 
artículos sobre cada periodo que contribuyen a la formación del panorama desde la antigüedad -por ejemplo, Haikal (2008) con su artículo Private collections and temple libraries in ancient Egypt o Dix (1994) con Public libraries in ancient Rome: ideology and reality- hasta documentos sobre la actividad en tiempos recientes como Keyser (2012) con Indexing: from thesauri to the semantic web o Chowdhury y Chowdhury (2007) en Organizing information: from the shelf to the web.

En el ámbito angloparlante es posible recuperar varios estudios diacrónicos sobre la organización temática de los recursos de información y decenas de trabajos sobre cada periodo. En el contexto latinoamericano es imprescindible una mayor investigación y producción literaria sobre la organización temática de los recursos de información, en general, y sobre su evolución histórica, en particular. Dicho lo anterior, esta investigación tiene por objetivo trazar una revisión diacrónica de la organización temática de los recursos de información en las bibliotecas, considerando los recursos, el proceso, las personas que lo desarrollan y los lenguajes de indización auxiliares de la actividad.

Para cumplir con el propósito establecido, el estudio se fundamentó en la revisión y análisis de un conjunto de fuentes de información relevantes sobre el tema. A partir del análisis desarrollado, el presente artículo presenta diacrónicamente etapas trascendentales por las que ha atravesado la organización temática de los recursos de información. Los resultados, se exponen en cuatro periodos: (1) antes del establecimiento de la bibliotecología como disciplina, (2) la estandarización bibliotecaria de los siglos XIX y XX, (3) los tiempos de la segunda mitad del siglo XX y (4) los albores del siglo XXI.

Es importante mencionar que la investigación se ofrece como un artículo de revisión que pueda ser de gran utilidad para la formación académica de estudiantes de bibliotecología, una obra de consulta para profesionales de la disciplina interesados en el tema y un material de lectura para todas aquellas personas apasionadas por la historia de las bibliotecas y sus procesos. 


\section{Desarrollo}

\section{a. El periodo antes del establecimiento de la bibliotecología como disciplina}

Nuestro conocimiento sobre el momento preciso en el que inició el tratamiento temático de los recursos de información es reducido "debido a que mucha de la evidencia ha sido destruida por los estragos del tiempo, la quema de libros y la fragilidad de los materiales en los que la información ha sido registrada" (Bates y Maack, 2010, p. 2282). No obstante, se cree que tal momento se encuentra asociado a épocas muy antiguas de la humanidad en las que surgieron recursos de información (huesos de animales, piezas de madera, tablas de arcilla, etc.) que superaron la capacidad de memoria humana. En cierto momento, "...alguien se dio cuenta de que los registros escritos debían organizarse para facilitar su acceso" (Cleveland y Cleveland, 2013, p. 33) y, justo entonces, el ser humano empezó a hacer algo para que la información almacenada fuera recuperable mediante el arreglo de ciertos rasgos en un orden establecido o a través de la condensación de documentos extensos en resúmenes o nociones elementales del tema tratado (Witty, 1973).

Las evidencias más antiguas sobre el tratamiento temático de recursos de información se encuentran en la civilización mesopotámica, alrededor del año 3000 a. C. (Stockwell, 2001; Gil Leiva, 2008; Cleveland y Cleveland, 2013). La civilización mesopotámica, formada por los pueblos sumerios, babilonios, acadios y asirios, se organizó en ciudades estado y desarrolló actividades políticas, sociales, económicas y religiosas. Como resultado, generaron información registrada en tablas de arcilla de diversos tamaños. Las primeras instituciones resguardadoras de documentos de las cuales se tienen pruebas fehacientes son "los repositorios de tabletas de arcilla de los Sumerios y las colecciones de cilindros de arcilla de los Asirios" (Stockwell, 2001, p. 30). Tales recursos, ocasionalmente, hacían referencia al contenido temático que albergaban; en las tablas los escribas solían agregar datos de contenido expresadas en resúmenes cortos, mediante oraciones breves o palabras referidas al contenido (Gil Leiva, 2008).

En Egipto, desde el año 3500 a. C. hasta el 30 a. C. se contó con depósitos de registros documentales tanto en el ámbito institucional como privado (Webb, 2013). Zinn señala que mientras las bibliotecas actuales buscan ofrecer servicios, las bibliotecas de ese periodo 
perseguían la "preservación y transmisión del saber colectivo" (2007, p. 175). Hacia el año 2000 a. C. las tablas de arcilla, como soporte de la información, fueron sustituidas por papiros que para enrollarse tenían una vara de soporte en la cual los encargados del depósito solían colgar etiquetas con las primeras frases del documento u oraciones relacionadas con el asunto tratado (Gil Leiva, 2008). Aunado a ello, algunos resquicios arqueológicos muestran inscripciones en los muros de los depósitos de documentos que registran información sobre los materiales albergados (Webb, 2013). Tales inscripciones no intentaban ser una clasificación o un catálogo bibliotecario, pero al paso del tiempo muestran posibilidades de una primigenia organización de las colecciones.

Las grandes bibliotecas de la antigüedad también desarrollaron actividades asociadas al tratamiento temático de sus materiales. En Hattusa, antigua capital del imperio hitita, se han encontrado restos de tablas de arcilla con información sobre conjuros, himnos, oraciones, asuntos médicos, registros históricos, leyes, y listas de vocabularios y "para mantener el orden en la biblioteca un número de tabletas eran catálogos de títulos" (Olof, 1998, p. 48). La biblioteca de Nínive, establecida por Ashurbanipal era vasta temáticamente, incluía obras sobre asuntos administrativos, legales, diplomáticos, comerciales, espirituales, encantamientos, himnos a dioses, tratados médicos, compendios astronómicos y trabajos literarios. Tenía más de doce salas y las tabletas estaban temáticamente organizadas, mientras un pequeño inventario se encontraba frente a cada sala (Polastrón, 2007; Borcoman, 2017). En La biblioteca de Ebla en el norte de Siria una misión arqueológica emprendida en 1974 encontró restos de más de 16,000 tabletas de arcilla que datan de aproximadamente el año 2400 a. C., y, en algunas de sus partes, se observan palabras referidas a entradas o secciones de la información registrada posteriormente (Cleveland y Cleveland, 2013).

Una biblioteca de la que se tiene más información sobre sus colecciones y organización fue la de Alejandría, fundada en 300 a. C. Es considerada la mayor biblioteca de la antigüedad y se cree que albergó aproximadamente 700 mil papiros (Borcoman, 2017). Los ptolomeos, que reinaron por casi trescientos años, promovieron reunir en ella colecciones amplias y hacerlas accesibles. Sus materiales fueron ordenados según su contenido temático y colocados en colecciones generales o específicas. Zenodoto (330 a. C.-260 a. C.) empleó el orden alfabético como modelo de organización (Cleveland y 
Cleveland, 2013); no obstante, "cuando hablamos de arreglo alfabético en la antigüedad, no significa el detallado letra-por-letra-hasta-el-final-de-la-palabra" (Witty, 1973, p.194), sino un orden más ligero que contemplaba la primera letra o acaso las tres primeras. Posteriormente, Calímaco (305-204 a. C.) generó el catálogo Pinakes formado por "120 rollos y contenía registros sobre los autores y sus obras" (Olense, 2014, p. 5). Se cree que el catálogo pudo haber tenido diez divisiones temáticas; sin embargo, solo tres son conocidas con certeza: leyes, retórica y miscelánea. También se piensa que pudo haber tenido "subclases de tipo cronológico, topográfico o biográfico" (Witty, 1958, p. 136).

En las culturas clásicas también se encuentran registros del tratamiento temático de los recursos de información (Polastron, 2007). En Grecia, cuna de la civilización occidental, los soportes de la información fueron tablas de diversos materiales (madera, arcilla, metal) y el pergamino en rollos de diversos tamaños. Las bibliotecas contaban con colecciones vastas, inventarios de los recursos y memorias de consultas (Oldfather, 1938). La biblioteca imperial, según Tucci, fue más allá del arreglo de la "división en secciones griegas y latinas (una distinción académica que es muy probable que esté equivocada)" (Tucci, 2013, p. 247). Es casi seguro que tenía colecciones temáticas o autorales y subcolecciones a partir de categorías semejantes creadas por los curadores de cada área.

En Roma el conocimiento fue un aspecto fundamental y "todas las casas de los hombres ricos tuvieron repositorios" (Stockwell, 2001, p. 32) que albergaban obras de filósofos, políticos, retóricos, poetas, etc. Entre las bibliotecas particulares destacaron las de Lucio Carpunio Piso, Lucinio, Marco Tulio Cicerón y Tito Pomponio Ático. Tales espacios estaban organizados, los documentos eran colocados en cajas de madera agrupadas bien por autor o contendido temático (Dix, 1994). En los años siguientes, la idea de tener bibliotecas públicas tomó fuerza y fueron establecidas la Bibliotheca in Atrio Libertatis, Bibliotheca Templi Apollinis, Bibliotheca Porticus Octaviae, Bibliotheca Templi Augusti, Bibliotheca Domus Tiberiana, Bibliotheca in Templo Pacis, Bibliotheca in Foro Traiano, Bibliotheca in Capitolio, Bibliotheca in Templo Aesculapi. Una encuesta de las construcciones romanas llevadas a cabo en el año $337 \mathrm{~d}$. C. indica la existencia de 28 bibliotecas públicas (Stockwell, 2001) reguladas por el Procurator Bibliotecorum. 
Durante la Edad Media, las bibliotecas estuvieron asociadas principalmente a las cortes y los monasterios. Aunque el periodo frecuentemente es considerado de oscurantismo, en lo relativo a las bibliotecas hubo avances. En el Scriptorium -zona específica de los conventos- los religiosos se organizaban para desempeñar cinco tareas: (1) la preparación de los pergaminos, (2) la copia de la obra, (3) la decoración del manuscrito, (4) la colocación y la gestión del manuscrito en la biblioteca y (5) la comercialización o distribución de copias para otras bibliotecas (Stockwell, 2001). El contenido temático tratado en los manuscritos fue central para su recuperación y el resumen del documento se empleó como una técnica para que los usuarios determinaran su utilidad.

Llegado el siglo XV, algunos hechos contribuyeron a una renovada concepción histórica: la caída de Constantinopla, la invención de la imprenta, el auge de las Universidades y los grandes descubrimientos científicos (Galende Díaz, 1996). La imprenta fue una innovación que posibilitó avances en todos los campos científicos y permitió a la disidencia religiosa florecer. Datos, pensamientos y expresiones que "pudieron rápidamente haberse perdido en el abismo de sistemas de memoria defectuosos, métodos de escritura engorrosos e inadecuadas formas de transportación ahora podían ser mantenidos por décadas entre las cubiertas de los libros" (Stockwell, 2001, p. 47). Las colecciones de las bibliotecas crecieron debido a la naciente facilidad de reproducción y, consecuentemente, durante los siglos XV y XVI los encargados de las bibliotecas se vieron en la necesidad de crear catálogos de consulta local y "ordenamientos de las colecciones por disciplinas" (O’Brien, 2016, p. 228).

Para el siglo XVII el avance de los impresos había crecido de forma impresionante, predominaban periódicos, revistas, novelas, panfletos, publicaciones para niños, etc. (Stockwell, 2001) y apareció un nuevo tipo de recurso de información: la revista (Cleveland y Cleveland, 2013). Con la proliferación de las revistas, los índices se volvieron esenciales. Al principio fueron instrumentos rudimentarios, formados de títulos y referencias a las páginas, pero pronto empezaron a combinar el acceso temático al final de la publicación. Justo hacia finales de ese periodo, tras el fortalecimiento del mundo académico y la naciente literatura científica, los índices y tareas de control temático de los recursos se vislumbraron necesarios $\mathrm{y}$, consecuentemente, los siglos posteriores se caracterizaron por la institucionalización de las bibliotecas, el surgimiento de la profesión bibliotecaria y la aparición de normas para la organización tanto descriptiva como temática de los recursos de información. 


\section{b. La estandarización de los siglos XIX y XX}

Durante el siglo XIX la organización tanto descriptiva como temática de los recursos de información se observó impostergable, en vista de que las bibliotecas albergaban gran cantidad de recursos (Mahapatra y Bhubaneswar, 1999). Según Stockwell (2001). El primer catálogo de libros por tema apareció en la biblioteca de la abadía de St. Galo en Suiza durante el siglo XIX y consistió en un registro calendárico-temático sobre las vidas de los santos y asuntos religiosos, de modo que fueran fácilmente localizables para su lectura en los días de festividad. Se formaba de categorías generales: la biblia, escritos de los padres de la iglesia, obras teológicas, homilías, vidas de santos y literatura secular.

Una figura icónica del siglo XIX en torno a la organización de los recursos de información fue Anthony Panizzi (1797-1879), director de la Biblioteca del Museo Británico. Su principal aporte a la bibliotecología reside en sus 91 reglas para la compilación del catálogo bibliotecario (1941) que tratan sobre el registro de los autores, los títulos y el ordenamiento alfabético del catálogo. Aunque la obra no considera asuntos relativos a la organización temática, abre la puerta a un nuevo periodo de estandarización bibliotecaria.

Charles Ami Cutter (1837-1903) fue otro personaje clave para el desarrollo del trabajo bibliotecario. Su obra Rules for a dictionary catalog en 1876 estableció un paradigma en la organización de recursos de información. Respecto a la organización temática, estableció dos proposiciones trascendentales: (1) permitir a las personas (no habla de usuarios) encontrar un libro cuyo tema es conocido y mostrar lo que la biblioteca tiene de un tema dado y (2) analizar asuntos relacionados con el lenguaje, los sinónimos, las palabras temáticas, los homónimos y los temas compuestos al representar temáticamente un material de la biblioteca (Cutter, 1891). Además de hablar de las palabras que pueden ser usadas como tema de los libros, esboza aspectos de normatividad y control que serán fundamentales para la categorización inherente a la representación temática de las colecciones y el desarrollo de vocabularios controlados.

Melvin Dewey (1851-1931) fue un bibliotecario esmerado en su profesión y relevante para la estandarización bibliotecaria. Como indica Lor, compartió "la preocupación de los bibliotecarios del siglo XIX por construir instituciones encargadas de adquirir, catalogar, acomodar y proporcionar acceso a las colecciones; aunado a ello, tuvo un constante 
énfasis en las cuestiones técnicas" (2019, p. 23). Dewey dirigió sus esfuerzos tanto al desarrollo de herramientas para la gestión de las colecciones bibliotecarias a través de la planeación y construcción del Sistema de Clasificación Decimal Dewey (1976) como para la formación de bibliotecarios profesionales mediante el establecimiento de la Columbia School of Library Economy en 1887. Esta última acción fortaleció la figura del bibliotecario y, en los años posteriores, será la base que apuntalará la instauración de la bibliotecología como una disciplina con actividades enfocadas a la gestión formal de los recursos de información.

Paul Outlet (1868-1944), frecuentemente denominado padre de las ciencias documentales es otra de las figuras representativas de nuestro campo de conocimiento. Durante su vida trabajó en la creación del Instituto Internacional de Bibliografía (1895), publicó el Tratado de documentación (1934), una obra fundamental sobre la profesión bibliotecaria y contribuyó para la consolidación de la Clasificación Decimal Universal, a partir de modificaciones sustanciales a la clasificación decimal elaborada por Melvin Dewey en 1876 (Arnaut, 1995).

En 1876 se establece la American Library Association (ALA), una organización profesional de bibliotecarios cuya misión fue congregarse "para permitir a los bibliotecarios hacer su trabajo más fácilmente y con menos gastos" (ALA, 2020). Posteriormente, su acta constitutiva de 1879 contempla una misión más amplia sobre su misión, asociada a:

[...] promover [los] intereses de las bibliotecas [del país] y de todo el mundo intercambiando opiniones, llegando a conclusiones e induciendo a la cooperación en todos los departamentos de la ciencia y la economía bibliotecaria; inclinar la voluntad de las personas para la fundación y el mejoramiento de las bibliotecas; y cultivar la buena voluntad entre sus propios miembros (ALA, 2020).

Derivado de la consolidación de la American Library Association, en 1895 apareció List of subject headings for use in dictionary catalogs, un apéndice a las reglas de Cutter para el catálogo diccionario cuyo objetivo fue simplificar el trabajo del catalogador en lo tocante a la representación temática de los recursos de información. En su construcción, la herramienta recuperó otros vocabularios controlados como la lista de temas de Peabody Institute y The harvard subject index (Cleveland y Cleveland, 2013). Incluía 
encabezamientos comunes para una biblioteca pequeña o mediana y daba algunas recomendaciones respecto a cómo construir un encabezamiento (forma, sintaxis, número) cuando no estuviese en la lista y fuese requerido.

Para este momento la Biblioteca de Congreso de los Estados Unidos se había establecido como una institución dirigida a la investigación y desarrollo de estándares bibliotecológicos. En lo tocante al contenido temático, desde 1898 trabajaron en la construcción de un listado autorizado y en 1909 publican Subject headings used in the library catalogues of the Library Congress. Rápidamente la herramienta obtiene reconocimiento y "se convierte en referente para el resto de las bibliotecas del mundo que la traducen o adoptan en otros idiomas" (Gil Leiva, 2008, p. 110). Como señala Chan (2020, párr. 3) "esta ahora enorme estructura comenzó con un pequeño paso. Después de que la biblioteca se trasladó del Capitolio a su nuevo edificio en 1897, sus funcionarios se enfrentaron a la pregunta de cómo debía organizarse la colección".

Para 1950 la organización temática de los recursos de información era una actividad común en las bibliotecas. Se fundamentaba en el análisis documental, la determinación del contenido temático de los materiales y su concreción en símbolos extraídos de vocabularios controlados, principalmente encabezamientos de materia. En cuanto al acceso temático, el catálogo era impreso y los materiales se encontraban físicamente ubicados en estanterías, según un sistema de clasificación.

\section{c. Los tiempos de la segunda parte del siglo XX}

Pasada la primera mitad del siglo, el periodo posguerra trajo consigo una gran explosión de la información basada en el uso de tecnologías computacionales (Cleveland y Cleveland, 2013). Las potencias mundiales emprendieron arduas labores de desarrollo tecnológico y hubo una creciente demanda de información bajo temáticas generales y altamente especializadas (Mahapatra y Bhubaneswar, 1999). "La investigación sobre IRR [Information Representation and Retrieval] no se transformó en un campo clave en las ciencias de la información hasta el estallido de la Segunda Guerra Mundial" (Chu, 2010, p. 1). A partir de entonces y durante todo el siglo $X X$ se hizo necesario recuperar información de modo preciso, en derivación, se mejoraron los procesos de selección, adquisición, almacenamiento, diseminación y recuperación de los recursos de información en bibliotecas gubernamentales, académicas y públicas. 
A mitad del siglo, ALA concreta un código de catalogación en el que habían estado trabajando desde 1908 y publica Rules for descriptive cataloging in the Library of Congress donde establece un área de descripción asignada al contenido temático de los recursos. Posteriormente, encarga a Seymour Lubetzky (1898-2003) la revisión de las normas y en 1967 aparece la primera edición de Anglo American Cataloguing Rules (AACR) que sigue manteniendo el área de descripción bibliográfica asociada a la representación del contenido temático de los recursos.

También a mitad de siglo, Hans Peter Luhn establece las bases de la indización automatizada. Propone, el índice KWIC (Keywords in Context-Palabras en contexto) el cual a partir de los términos más frecuentes del documento establece de qué trata temáticamente. La indización automatizada se observó como una opción viable para el tratamiento temático de los recursos de información en vista de que eliminaba la subjetividad inherente al proceso de catalogación humana y reducía costos y tiempo de indización; sin embargo, también evidenció que la computadora no posee el poder analítico de los humanos (Gil-Leiva y Rodríguez-Muñoz, 1997).

En la década de 1960 las metodologías para la construcción de tesauros empezaron a desarrollarse y "alcanzaron su momento de mayor esplendor en 1980's cuando alcanzaron el estatus de lineamientos ISO para tesauros monolingües y para tesauros multilingües" (Keyser, 2012, p. 7). El tesauro tiene sus antecedentes en el siglo XII, periodo en el que fueron elaborados compendios denominados Thesauri que eran "obras conceptuales de control sinonímico y bases de datos léxicas". (Bates y Maack, 2010, p. 2283). En comparación, los tesauros como herramientas documentales son un asunto moderno enfocado al control del vocabulario de un área de especialidad. En 1967 se publica Guidelines for the development of information retrieval thesauri elaboradas por el Federal Council for Science Technology. En 1970 Guidelines for the stablishment and development of monolingual scientific and technical a cargo de la UNESCO, en 1974 ISO 2788: 1974 Guidelines for the stablishment of development of monolingual thesaun y en 1986 ISO 2788-1986 Guidelines for the stablishment and development of monolingual tesauri.

Los estándares antes mencionados "datan de un tiempo cuando la información era casi exclusivamente publicada en libros impresos y revistas que eran almacenadas en las 
bibliotecas físicas" (Keyser, 2012, p. 7); no obstante, tras la masificación de la web, el tesauro ha sido un lenguaje de indización que logró integrarse al mundo digital, en donde sus metodologías de planeación se han renovado con las nuevas normas ISO-259641:2011 Information and documentation-thesauri and interoperability with other vocabulariesPart 1: thesauri for information retrieval e ISO-25964-2:2013 Information and documentation-thesauri and interoperability with other vocabularies-Part 2: interoperability with other vocabularies, así como su correspondencia europea UNE-ISO 25964-1: 2014 Información y documentación. Tesauros e interoperabilidad con otros vocabularios. Parte 1: tesauros para la recuperación de la información y UNE-ISO 25964-2: 2016 Información y documentación. Tesauros e interoperabilidad con otros vocabularios. Parte 2: interoperabilidad con otros vocabularios.

Durante la década de 1980 hubo gran atención hacia la representación temática y el proceso que los indizadores realizaban para determinar el aboutness de un recurso de información (Hjørland, 1997). En 1981 se publica ISO-5127/3A-1981 Information and documentation vocabulary. Acquisition, identification and análisis of document and data que trata sobre la terminología asociada a la representación temática de los recursos de información. Luego, en 1985 se publica ISO 5963:1985 Methods fo examining documents. Determining their subjects and selecting indexing terms que, por una parte, define los lineamientos sobre el proceso que el catalogador debe seguir para establecer de qué trata un recurso $\mathrm{y}$, por otro, proporciona la pauta para la selección adecuada de los encabezamientos de materia o descriptores que simbolicen el contenido temático.

A lo largo de las décadas de los sesentas, setentas y ochentas, la bibliotecología enfrentó cambios que transformaron completamente su dominio de acción. Primero, a los libros y revistas impresos se agregaron otros soportes informativos como casetes, videos, discos compactos, etc. Luego, los catálogos físicos se convirtieron legibles por máquinas mediante formatos específicos como Machine Readable Cataloging (MARC). Por último, la década de los noventas se caracterizó por el catálogo público de acceso en línea (Online Public Access Catalog-OPAC) y la masificación de la web que redefinió el ciclo de la información y su consecuente recuperación, "dando paso al advenimiento de la computadora, el Internet y otras tecnologías de la información de la era digital y la era en red" (Chu, 2010. p. 4). 
Durante la primera mitad del siglo XX, la bibliotecología contó con pocas tecnologías para la producción, almacenamiento y ordenamiento de la información. En comparación, las décadas de la segunda mitad del siglo "representan los años dorados en el desarrollo de la representación y recuperación de información" (Chu, 2010, p. 2). Los cincuentas trajeron consigo el primer sistema de recuperación por computadora; los sesentas implicaron trabajos experimentales en la organización y en la recuperación temática, los setentas nos dieron una conversión generalizada para operaciones automatizadas, en los ochentas el tratamiento temático de los recursos de información migró hacia bases de datos y en los noventas nos llevaron al espacio web. De manera que:

Para cuando el siglo XX había terminado, era evidente que la indización había sobrepasado las ideas de los tiempos antecedentes. Habíamos progresado de los índices para obras monográficas, a los índices cooperativos, a los índices de bases de datos masivos y ahora teníamos la World Wide Web con muchos cambios formidables y oportunidades en la indización (Cleveland y Cleveland, 2013, p. 40).

Durante la segunda mitad del siglo XX se fortalecieron los estándares para la organización temática de la información y los lenguajes de indización. Por demás, la figura del bibliotecario se consolidó mediante el establecimiento de escuelas de bibliotecología en Estados Unidos, Europa y América Latina.

En nuestro contexto latinoamericano, aparecieron también algunas herramientas auxiliares de la organización temática de los recursos de información como Lista de encabezamientos de materia para bibliotecas (1967) compilada por Carmen Rovira y Jorge Aguayo, Lista de encabezamientos de materia (1978) de Gloria Escamilla y Lista de encabezamientos de materia para bibliotecas (1998) editada por la Biblioteca Luis Ángel Arango. En cuanto a la formación bibliotecológica se establecieron escuelas de bibliotecología en varios países de la región como México, Colombia, Costa Rica, Argentina, Uruguay y Cuba donde, además, se han establecido centros de investigación que cuentan con numerosas líneas de investigación propias de la disciplina. 


\section{d. Los albores del siglo XXI}

Derivado del surgimiento de nuevos soportes de la información y las nuevas necesidades de los usuarios, el arreglo temático de los recursos de información desde la perspectiva bibliotecológica tuvo una etapa de enriquecimiento, incorporando nuevas teorías y métodos (Peña Vera, 2011). Los trabajadores de las bibliotecas también aprendieron una nueva forma de actuar, derivada de las comunicaciones, la Internet y las tecnologías de la información. "El bibliotecario ahora es un especialista de la información y la biblioteca un centro de información" (Stockwell, 2001, p. 148). En este nuevo rol, los expertos del campo se mueven, por un lado, en el espacio físico de la biblioteca tradicional y, por otro, se insertan en el ciberespacio que se vislumbra como el nuevo mundo para repositorios y bibliotecas digitales (Rubin, 2010).

Llegados a este punto, fue indispensable reflexionar sobre un planteamiento esencial: ¿la organización temática de los recursos de información en los contextos bibliotecológicos digitales es distinta a la organización temática realizada en la biblioteca física? Al respecto, Martínez Arellano indica que:

Los tipos de acceso temático no han sido modificados substancialmente, a pesar de haber surgido nuevas opciones para la recuperación temática apoyada en alternativas que actualmente brindan los sistemas y las tecnologías, como son los thesaurus, los sistemas de indización, las ontologías y la web semántica (Martínez, 2012, p.7.)

Como señala Martínez Arellano, el acceso temático no ha sido modificado sustancialmente, pero a partir de las posibilidades tecnológicas, el abanico de los sistemas para la representación y organización temática de los materiales ha crecido. Chowdhury y Chowdhury (2007) coinciden con tal aseveración cuando asumen que:

Con la introducción de la Web y las bibliotecas digitales, el mundo de la información ha cambiado de manera dramática en la última década en términos de volumen, variedad, formato, naturaleza y complejidad de recursos de información electrónica. Esto dio lugar a la necesidad de nuevas y mejores herramientas, técnicas y estándares para la organización de la información (Chowdhury y Chowdhury, 2007, p.18). 
La organización temática de los recursos de la información actualmente se desarrolla mediante procesos manuales, automatizados o híbridos (Gil Leiva, 2008).

a) La indización manual se fundamenta en la tarea experta de un indizador quien aplica un proceso de análisis documental y a través de la consulta de lenguajes de indización (tesauros, taxonomías, encabezamientos de materias, etc.) concreta en signos lingüísticos qué tratan los recursos.

b) La indización automatizada emplea programas que, mediante frecuencias estadísticas y algoritmos lingüísticos, establecen de qué tratan temáticamente los recursos de información.

c) La indización híbrida se vale de tecnologías que pueden sugerir los temas del recurso a la vez que hay validaciones humanas sobre los encabezamientos o descriptores propuestos.

En cuanto a las normas, los cambios derivados de las tecnologías trajeron consigo modelos conceptuales acordes al siglo XXI, entre los que es posible mencionar FRBRFuncional Requeriments for Bibliographic Records y su derivación sobre el control de autoridades temáticas en FRSAD-Functional Requeriments for Subject Authority Data que plantea aspectos concretos sobre cómo debe ser el acceso temático actualmente (IFLA, 2010).

Aunque la organización temática de los recursos de información no ha sido modificada sustancialmente, han surgido nuevas normas y lenguajes de indización, también denominados sistemas para la organización del conocimiento (Hodge, 2000). Los lenguajes de indización tradicionales (encabezamientos de materia, tesauros) se han enriquecido con nuevas estructuras como tesauros y taxonomías digitales que han migrado al espacio web y mapas tópicos, ontologías y folksonomías emergidas del mismo contexto digital (Suárez Sánchez, 2017). Al respecto Keyser (2012, p. 21) señala que por más de un siglo las bibliotecas han estado trabajando con clasificaciones, encabezamientos de materias y tesauros, pero "nuevos instrumentos para clasificar e indizar documentos y el conocimiento fueron inventadas en el mundo de la gestión del conocimiento: taxonomías y ontologías" que abren las posibilidades de la organización temática hacia tiempos actuales y futuros vinculados con el ciberespacio. 
Actualmente, la organización temática de los recursos de información en las bibliotecas considera dos aspectos: la teoría bibliotecológica más sustancial sobre representación, organización y recuperación de la información y, por otro, agregó componentes tecnológicos. Retomo a Hodge cuando señala que:

La tecnología facilita la conexión de comunidades de conocimiento dispares a nivel de red. Sin embargo, el descubrimiento de los recursos y la verdadera accesibilidad requieren que el contenido y su organización sean comprendidos por estas comunidades dispares. Al proporcionar accesos temáticos alternativos, añadir modos de comprensión, apoyar el acceso multilingüe y proporcionar términos para ampliar la búsqueda de texto libre, los KOS pueden facilitar el descubrimiento y la comprensión por parte de comunidades dispares, y permitir que estas comunidades interactúen de nuevas maneras (Hodge, 2000, p. 22).

Las actividades bibliotecarias respecto a la organización temática de los recursos en el siglo XXI se mantienen vigentes y en continuidad. Asimismo, el acceso temático a los recursos deberá mantenerse ya sea mediante herramientas que generen entradas alfabéticas o sistemas más tecnológicos que posibiliten estructuras jerárquicas o semánticas (Hider y Harvey, 2008).

\section{Conclusiones}

La historia de la organización temática de los recursos de la información en las bibliotecas es extensa y evolutiva como la sociedad misma. Como señala Peña Vera (2011), la relación entre las necesidades recuperadoras y la actividad organizadora ha sido la constante en la evolución de la organización temática de los materiales a lo largo del tiempo. En la actividad se encontraron implicados cuatro elementos: (1) los recursos de información, (2) las necesidades de los usuarios, (3) el procedimiento ordenador y (4) los lenguajes de indización. Todos son dependientes, pues en la medida en que los soportes de la información y las necesidades de las personas usuarias cambian, el proceso y las herramientas deberán ajustarse para dar respuesta al arreglo (Chowdhury y Chowdhury, 2007). 
Derivado del objetivo inicial del estudio: trazar una revisión diacrónica de la indización considerando los recursos, el proceso, las personas que la realizan y las herramientas auxiliares de la actividad, se identificó que la organización temática de los recursos de información ha sido una actividad constante que abarca desde las civilizaciones más antiguas (Mesopotamia, Egipto, Grecia, Roma), atraviesa períodos históricos esenciales de la civilización occidental (Edad Media, Renacimiento, Edad Moderna) y se mantiene vigente en los tiempos actuales.

Los resultados obtenidos ofrecen un somero análisis de la organización temática de los recursos de información a lo largo del tiempo a la vez que evidencian momentos, personajes, estándares y lenguajes de indización que requieren ser investigados con mayor detenimiento y análisis. En consecuencia, el presente trabajo abre un inmenso abanico de líneas de investigación sobre una etapa específica del ordenamiento temático de las entidades bibliográficas (Antigüedad, Edad Media, Renacimiento, siglo XX, etc.), trabajos comparativos entre una etapa y otra, estudios evolutivos de las normas y herramientas auxiliares de la actividad (vocabularios controlados, reglas de catalogación) y más trabajos panorámicos sobre el tema en lengua española.

De igual modo, es indispensable trazar un panorama diacrónico de la organización temática de los recursos de información en el contexto latinoamericano que, indudablemente, tendrá múltiples variantes en comparación con una visión general en la que prevalecen procesos y herramientas del contexto anglosajón.

Finalmente, es importante mencionar la relevancia de la organización temática de los recursos de información y el trabajo de los bibliotecarios quienes mediante la actividad tiende un puente entre las necesidades tópicas de la población usuaria y los recursos que la satisfagan. Dado que la biblioteca es una institución en constante evolución, cada una de sus actividades lo son y la organización temática de los recursos de información no es la excepción. En los tiempos antiguos empezó a modelarse, durante el siglo XX se estandarizó y actualmente posibilita el acceso tópico tanto en catálogos bibliotecarios asociados con la biblioteca física como en sistemas recuperadores de información vinculados con la biblioteca digital. Así, su tendencia, es hacia la continuidad como actividad y tema de investigación con ricas vetas de aprendizaje y conocimiento. 


\section{Referencias}

ALA. (2020). American Library Associatión (ALA). Recuperado de http://www.ala.org/ Arnau, P. (1995). Trascendencia de la vida y obra de Paul Otlet. Revista General de Información y Documentación, 5(2), 153-162.

Barité, M. (2015) Diccionario de organización del conocimiento: Clasificación, indización, terminología. Montevideo: Universidad de la República de Uruguay.

Bates, M. y Maack, M.N. (ed). (2010) Encyclopedia of library and information sciences. $3^{\text {a }}$ ed. Boca Ratón, Florida: CRC.

Borcoman, M. (2017). Niniveh and Alexandria: the two vanished libraries of antiquity. Romanian Journal of Library and Information Science. 13, 109-112.

Chan, L.M. (2020). Still robust at 100. A century of LC subject headings. Recuperado de https://www.loc.gov/loc/lcib/9808/lcsh-100.html.

Chowdhury, G. y Chowdhury, S. (2007). Organizing information: from the shelf to the web. Londres: Facet Publishing.

Chu, H. (2010) Information representation and retrieval in the digital age. Medford, New Jersey: Information Today.

Cleveland, D. y Cleveland, A. (2013). Introduction to indexing and abstracting. Santa Barbara, California: Libraries Unlimited.

Cutter, Ch. (1891). Rules for a dictionary catalogue. Washington: Goverment Printing Office.

Dix, T. (1994). "Public libraries" in Ancient Rome: ideology and reality. Libraries \& Culture. 29(3), 282-296.

Galende Díaz, J. (1996). Las bibliotecas de los humanistas y el renacimiento. Revista general de información y documentación, 6 (2), 91-123.

Gil-Leiva, I. (2008). Manual de indización: teoría y Práctica. Gijón: TREA.

Gil-Leiva, I. y Rodríguez-Muñoz, J. (1997). De la indización humana a la indización automática. En García Marco, F. J. Organización del conocimiento en sistemas de información y documentación: actas del II Encuentro de ISKO-España (201-215). Zaragoza, España: Universidad de Zaragoza.

Haikal. F. (2008). Private collections and temple libraries in ancient Egypt. En El-Abbadi, M., ¿ed. What happened to the ancient library of Alexandria? (39-54). Leiden: Brill. 
Hider, P y Harvey, R. (2008). Organizing knowledge in a global society: principles and practices in libraries and information centres. Australia: Centre for Information Studies.

Hjørland, B. (1997). Information seeking and subject representation: an activity-theoretical approach to information science. Westport, Connecticut: Greenwood.

Hodge, G. (2000). Systems of knowledge for digital libraries: beyond traditional authority files. Washington: Council on Library and Information Resources.

IFLA. (2010). Functional Requirements for Subject Authority Data (FRSAD). Recuperado de https://www.ifla.org/files/assets/classification-and-indexing/functional-requirementsfor-subject-authority-data/frsad-final-report.pdf.

Keyser, P. (2012). Indexing: from thesauri to the semantic web. Oxford, Reino Unido: Chandos Publishing.

Lor, P. (2019). What were librarians doing while Otlet was inventing documentation? the modernization and professionalization of librarianship during the Belle Époque. Italian Journal library. Archives and Information Sciencie. 10(3), 18-33.

Mahapatra, P. y Bhubaneswar, Ch. (1999). Organizing information in libraries. Nueva Delhi: Ess Ess.

Martínez, F. (2012). Retos de la catalogación y los catálogos. Investigación Bibliotecológica, 26(58), 7-12.

O'Brien, J. (2016). Epilogue: medieval libraries in the sixteenth century: a dream of order and knowledge. French Studies: A Quarterly Review, 70(2), 228-238.

Oldfather, W.A. (1938). The maintenance of ancient Greek public libraries. The Library Quarterly: Information, Community, Policy, 8(2), 287-288.

Olesen Bagneux, O. (2014). The memory library: how the library in hellenistic Alexandria worked. Knowledge Organization, 41(1), 3-13.

Olof, P. (1998). Archives and libraries in the ancient near east 1500-300 B. C. Bethesa, Maryland: CDL Press.

Peña Vera, T. (2011). Organización y representación del conocimiento: incidencias de la tecnología de información y comunicación. Buenos Aires: Alfagrama.

Polastron, L. (2007). Libros en llamas. Historia de la interminable destrucción de bibliotecas. México: Fondo de Cultura Económica.

Rubin, R. (2010). Foundations of library and information science. New York: NealSchuman. 
Stockwell, F. (2001). History of information storage and retrieval. Jefferson, California del Norte: McFarland \& Company.

Suárez Sánchez, A. (2017). Sistemas para la organización del conocimiento: definición y evolución histórica. E-Ciencias de la Información, 7(2), 1-19.

Tucci, P. (2013). Galen and the library at antium: the state of the question. Classical Philology, 108(3), 240-251.

Webb, K. (2013). The house of books: libraries and archives in Ancient Egypt. Libri, 63(1), 21-32.

Witty, F. (1973). The beginnings of indexing and abstracting: some notes towards a history of indexing and abstracting in antiquity and the middle ages. The indexer, 8(1), 193198.

Witty, F. (1958). The Pínakes of Callimachus. Library Quarterly, 28(2), 132-136.

Zinn, K. (2007). Libraries and archives: the organization of collective wisdom in ancient Egypt. En Cannata, M, ed. Current research in egyptology 2006: proceedings of the 7th annual symposium. Oxford: University of Oxford.

\section{Notas de la Autora}

Adriana Suárez Sánchez. Instituto de Investigaciones Bibliotecológicas y de la Información, Universidad Nacional Autónoma de México, México. Investigadora Asociada. Correo electrónico asuarez@iibi.unam.mx. ORCID: http://orcid.org/0000-0003-0215-337X 